\title{
OPTICAL AND MAGNETIC SIGNATURES OF LOCALIZED EXCITATIONS
} IN POLYANILINE

W. P. SU

Department of Physics, University of Houston, Houston, Texas 77204-5506 ${ }^{1}$

and Department of Physics, The Ohio State University, Columbus, Ohio 43210-1106

A. J. EPSTEIN

Department of Physics and Department of Chemistry

The Ohio State University, Columbus, Ohio 43210-1106

J. KIM

Department of Physics, University of Houston, Houston, Texas 77204-5506

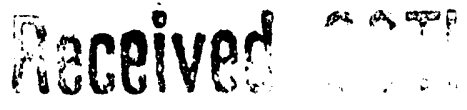

OCT 261998

\section{ABSTRACT}

Using a tight-binding Hamiltonian we study the lattice configurations and electronic structures of three forms of polyaniline: leucoemeraldine base (LEB), emeraldine base (EB) and pernigraniline base (PNB). Both bond length and ring rotation angle are considered in the structural relaxation. The dominant elementary excitations of isolated chains are found to be solitons in PNB and bipolarons in EB and in LEB. Optical absorptions of the ground states and of the solitonic and polaronic excitations are calculated and compared with experiments. The spin density profile of a single neutral soliton in PNB is discussed in connection with magnetic measurements.

Polyaniline is an interesting material due to its unusual transport, magnetic, optical and environmental properties. The simultaneous presence of the heteroatoms (the nitrogens) and the phenyl rings makes the theoretical modelling much less straightforward than that for polyacetylene. In this paper we show that it is nonetheless possible to understand much of the optical and magnetic properties from an SSH(Su-Schrieffer-Heeger) type Hamiltonian[1]. This allows a direct identification of the ground state as well as excited state lattice configurations of various forms of polyaniline.

We first discuss PNB. Ignoring the ring rotation for a moment, we can write down the following SSH-like Hamiltonian

$$
H=\sum_{\langle i, j\rangle}\left\{-\left(t_{0}-\alpha \delta r_{i j}\right) \sum_{\sigma}\left[c_{i, \sigma}^{\dagger} c_{j, \sigma}+H . c .\right]+\frac{K}{2}\left(\delta r_{i j}\right)^{2}\right\}+V_{0} \sum_{n, \sigma}^{1} c_{n, \sigma}^{\dagger} c_{n, \sigma} .
$$

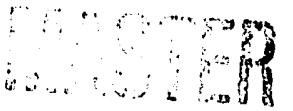

${ }^{1}$ Present and permanent address. 
Here $c_{i, \sigma}^{\dagger}$ creates an electron with spin polarization $\sigma$ in an atomic $2 p_{z}$ orbital on the $i$-th backbone atom (carbon or nitrogen). $j$ is a next neighbor of $i$, and the symbol $\langle i, j\rangle$ denotes summation over bond sites. $\alpha \delta r_{i j}$ is the modulation in interatomic hopping integral due to a change in bond length $\delta r_{i j}$ relative to a uniform refernce bond length. The spring constant $K$ determines the elastic energy of the sigma bonds. For simplicity we treat a carbonnitrogen bond like a carbon-carbon bond, but we do add the last term in (1) to reflect a distinct on-site potential $V_{0}$ on the nitrogen atoms. The prime in the last summation sign means a restricted summation over the nitrogen atoms only. $K^{\prime}=K / \alpha^{2}=1 / \mathrm{eV}$ and $V_{0}=-2 \mathrm{eV}[2]$ have been adopted in our calculations.

By minimizing the total energy with respect to $\delta t_{i j}=\alpha \delta r_{i j}$ one finds bond alternation. In addition to that, there is a uniform bond contraction during the relaxation from the $\delta t_{i j}=0$ initial configuration. A bare value of $t_{0}=1.6 \mathrm{eV}$ leads to an average renormalized hopping integral around $2.7 \mathrm{eV}$.

Besides the charged solitonic and polaronic states previously discussed by dos Santos and Brédas[3], we have also studied the relaxed configuration of an electron-hole pair initially photogenerated in a neutral pernigraniline sample. It corresponds to two well-separated kinks. The two gap states associated with the kinks are almost degenerate. Each of them is singly occupied. One can prove that in the adiabatic approximation those neutral spin solitons are directly photogenerated in contrast to polyacetylene wherein only charged solitons are directly photogenerated[4].

The energy gap thus calculated for PNB turns out to be about $1 \mathrm{eV}$, too small to account for the observed $2.4 \mathrm{eV}$ energy gap[5]. Hence we must examine the contribution of the ring rotational degree of freedom $[2,6]$. Let $\phi_{k}$ be the torsional angle of the $\mathrm{k}$-th phenyl-ring away from the $\mathrm{C}-\mathrm{N}-\mathrm{C}$ plane. Since steric repulsion favors ring twists of opposite signs at neighboring rings, as confirmed by $\mathrm{X}$-ray diffraction experiment[7], it is more useful to define the staggered order parameter $\psi_{k}=(-1)^{k} \phi_{k}$. Following Ginder and Epstein[2] we adopt the following form of the steric potential

$$
V_{s}=\sum_{k}\left[V_{11}\left(\sin \psi_{k}-\sin \psi_{k-1}\right)^{2}-V_{20} \sin ^{2} \psi_{k}+V_{40} \sin ^{4} \psi_{k}\right]
$$

The ring rotation decreases the overlap between the two $p_{z}$ orbitals of the C-N bond. Effectively, the hopping integral of that bond $-\left(t_{0}-\delta t_{i j}\right)$ gets multiplied by $\cos \psi_{k}$. This together with the addition of the steric potential (2) to (1) completes the inclusion of the ring rotation in the Hamiltonian. Henceforth we will refer to this Hamiltonian instead of the one in (1).

For PNB, three parameters $V_{11}, V_{20}$ and $V_{40}$ in (2) are taken to be $0.185 \mathrm{eV}, 4.5 \mathrm{eV}$ and $2.5 \mathrm{eV}$ respectively. They are chosen to reproduce the correct spin density distribution of the neutral solitons and to yield the proper Peierls gap. As in the pure bond order case, there are two degenerate ground states. Each one has a distinct ring rotation dimerization pattern with a uniquely determined bond alternation pattern. The ring that rotates further $\left(53^{\circ}\right)$ from the 
$\mathrm{C}-\mathrm{N}-\mathrm{C}$ plane is always benzenoid-like, while the quinoid-like ring twists a little $\left(12^{\circ}\right)$ from the C-N-C plane. This confirms the chemical intuition that it is harder to rotate double bonds than single bonds.

The optical absorbance calculated for a PNB chain in the ground state is displayed as the solid line in Fig. 1. The dashed curve depicts the absorption spectrum of the same PNB chain with a relaxed neutral soliton-antisoliton pair. The energy level diagram is also shown. As in the case of pure bond order soliton, the gap states associated with neutral soliton pairs $\left(\mathbf{S}^{0}, \overline{\mathbf{S}}^{0}\right)$ are almost degenerate. The effective charges of these spin $1 / 2$ neutral solitons are vanishingly small. The present model predicts two photoinduced absorption peaks at about $0.9 \mathrm{eV}$ and $1.5 \mathrm{eV}$ since the neutral soliton level is not at midgap due to the absence of charge conjugation symmetry. Just as for the absorption of neutral solitons in polyacetylene we expect that the subgap absorption to be Coulomb shifted by several tenths of an $\mathrm{eV}[8]$. These neutral soliton predictions are in agreement with the long time component of the photoinduced absorption data obtained by Coplin et al.[9], which shows that this shift is about $0.5 \mathrm{eV}$ (to $1.5 \mathrm{eV}$ and $1.8 \mathrm{eV}$ ). This identification is strengthened by the near absence of infrared active vibrational mode intensity associated with the $\mathbf{S}^{0}$ and $\bar{S}^{0}$ absorptions (reflecting essentially zero charge associated with these defects[9]) and electron paramagnetic resonance studies of PNB powders and solutions showing localized spins[10, 11].

To gain more insight into the neutral solitons, we have sketched the chemical structure of a neutral soliton $\mathbf{S}^{0}$ in Fig. 2. It is noted that neutral solitons that are centered on two adjacent quinoid-like rings are unstable and decay into the benzenoid type soliton described here. That explains why only one type of spin density distribution has been detected in neutral PNB

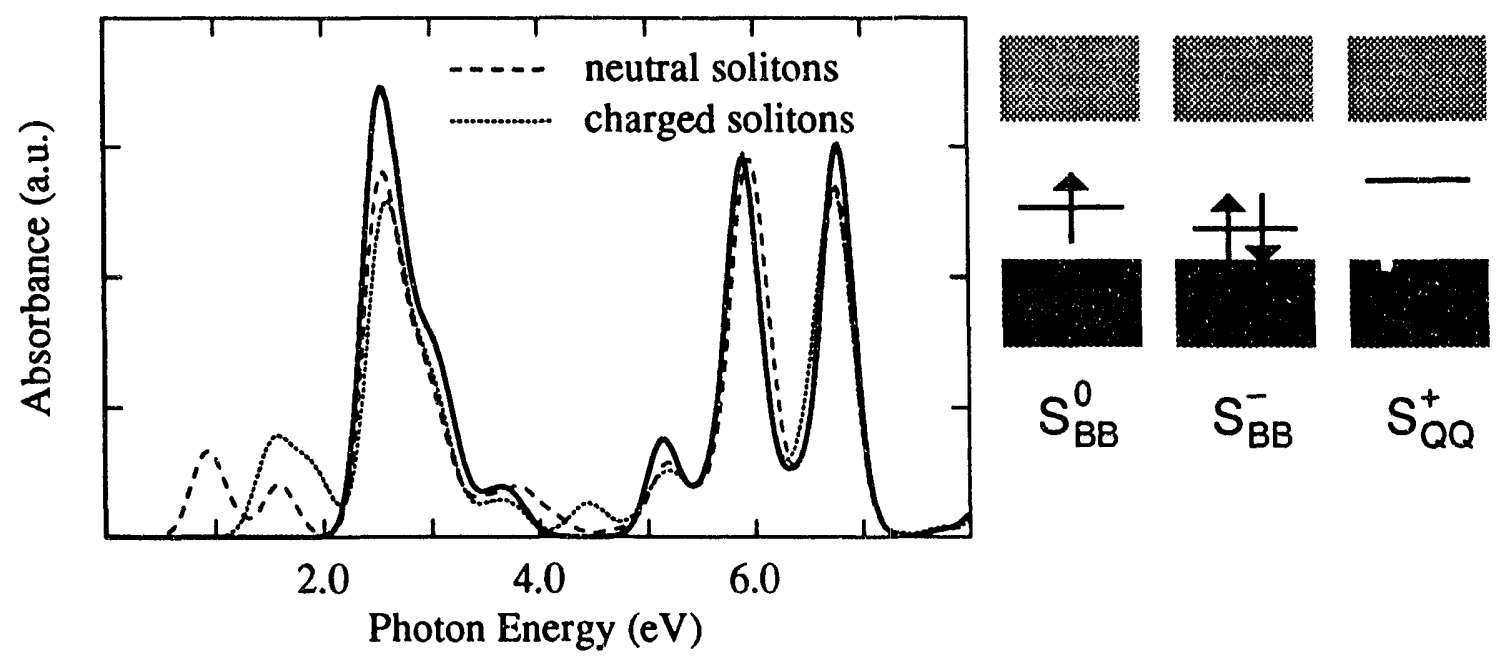

Fig. 1. Calculated optical absorbance of a PNB chain with a pair of solitons.

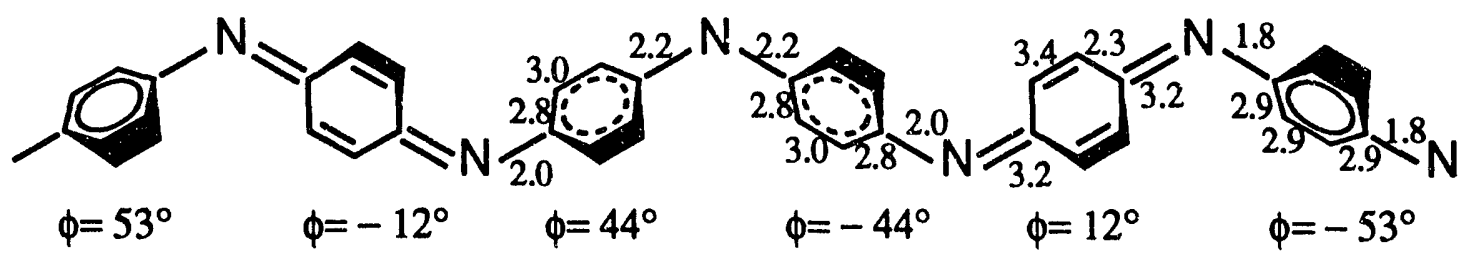

Fig. 2. Bond orcuers and torsional angles in the vicinitv of a neutral soliton in PNR 
samples $[10,11]$. The probability of finding an unpaired spin at the single central nitrogen site turned out to be 0.45 , which is comparable to the value 0.6 deduced from the ESR measurement by Cromack et al.[10] and Long et al.[11].

As opposed to neutral solitons, there are two distinct geometrical structures corresponding to the two charged states of charged solitons. The absorption spectrum of the PNB chain with an oppositely charged soliton pair is shown as the dotted line in Fig. 1. An $\mathrm{S}^{-}$consists essentially of two back-to-back benzenoid rings or $S_{B B}^{-}$, even more strongly localized than an $\mathrm{S}_{\mathrm{BB}}^{0}$. On the other hand, an $\mathrm{S}^{+}$resembles two adjacent quinoids, or an $\mathrm{S}_{\mathrm{Q} Q}^{+}$. The gap state wave function of an $\mathrm{S}_{\overline{B B}}^{-}$is similar to that of an $\mathrm{S}_{\mathrm{BB}}^{0}$, whereas there is a node on the central nitrogen site in the wave function of the gap state of an $S_{Q Q}^{+}$. As in polyacetylene, both charged solitons are spinless. The calculated subgap absorption in Fig. 1 can indeed account for the short time component of the photoinduced absorption measured by Leng et al.[12], provided that a Coulomb downshift of $0.5 \mathrm{eV}$ is considered.

Experimentally Leng et al.[12] and Coplin et al.[9] have recently examined the long time component of the photoinduced absorption in more details and have found two absorption peaks near $1.5 \mathrm{eV}$ and $1.8 \mathrm{eV}$. It is very tempting to associate them with the double peak feature seen in Fig. 1. The two peaks, however, do seem to have different dynamics which vary with temperature and laser intensity.

Let us now turn to LEB. There is no driving force for dimerization in LEB, but tine alternate ring flippings persist because the steric repulsions are still there. The ground states are doubly degenerate as in PNB. In principle ring-torsional kinks can exist as suggested and studied by MacKenzie et al.[13]. In practice, however, they are hard to excite. We find, for example, that two band electrons near the conduction band edge relax into a bipolaron and not a
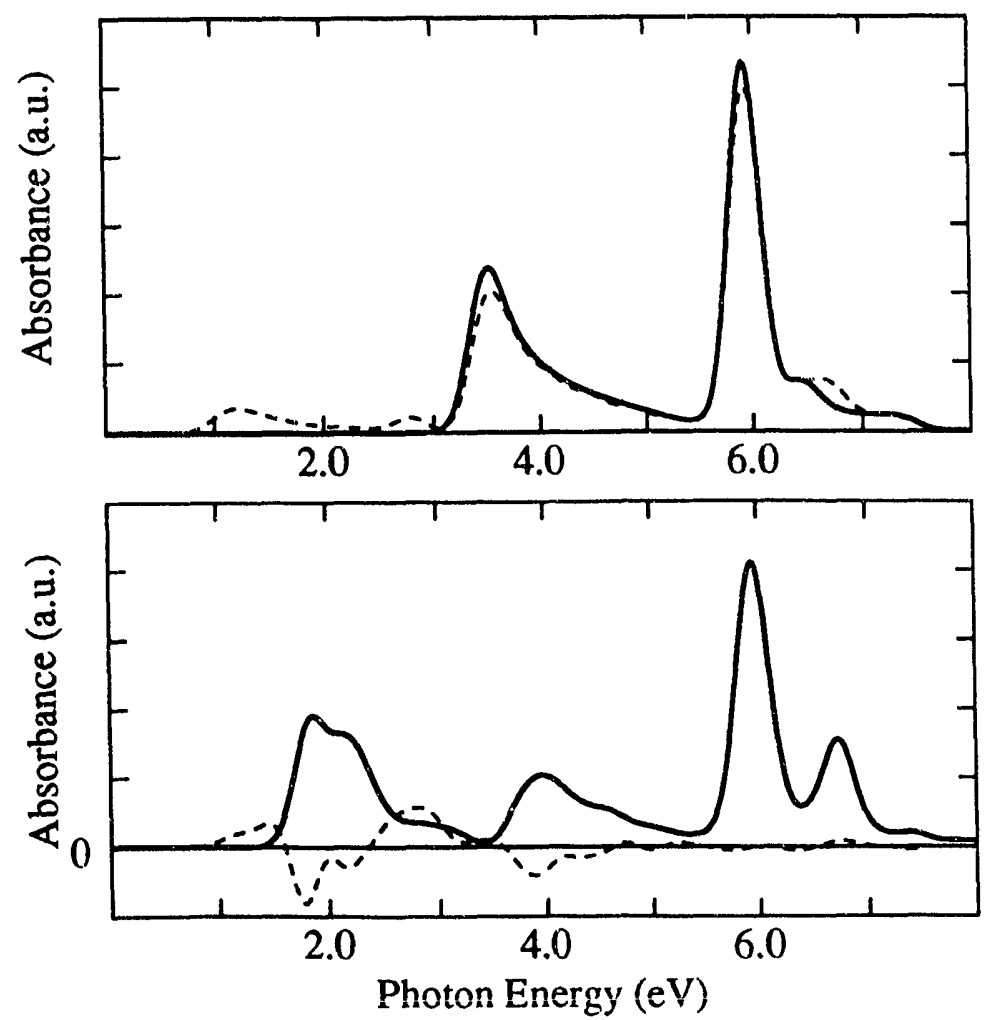

Fig. 3. Calculated absorption of an LEB chain in the ground state (solid line), with a hole bipolaron (dashed line).

Fig. 4. Calculated absorption of an EB chain in the ground state (solid line), and the photoinduced absorption of a bipolaron pair (dashed line). 
kink-antikink pair. As a generic feature of many electron-phonon systems, in the absence of Coulomb repulsion bipolarons here are more stable than polarons. For the model used here the absorption profile of a bipolaron appears to fit the photoinduced absorption of LEB better than those of polarons. Thus we suggest that bipolarons should be considered for the elementary excitations[14] in optical excitations in LEB.

It should be pointed out the we can not inherit all the parameter values in the Hamiltonian from PNB. We have simply adopted a smaller value of $V_{20}=3.0 \mathrm{eV}$, which leads to a uniform $\psi_{n}=37^{\circ}$. The calculated absorbance of the ground state of LEB shown in Fig. 3 compares favorably with existing experimental data[15]. An electron bipolaron gives rise to very small subgap absorption compared to a hole bipolaron as depicted in Fig. 3. This is consistent with the reasoning of McCall et al.[15]. Another important feature of the bipolaron absorption is that the lowest absorption peak is more visible than the one at a higher energy. Within this model, the lowest photoinduced absorption peak of $\mathrm{McCall}$ et al. at about $0.6 \mathrm{eV}$ and part of the $3.0 \mathrm{eV}$ PA peak can be interpreted as intrinsic to LEB. The rest of the spectroscopic features can be ascribed to residual emeraldine base segments contained in LEB. The difference between the calculated peak energy in Fig. 3 of about $1.1 \mathrm{eV}$ and the experimental $0.6 \mathrm{eV}$ can again be regarded as a Coulomb shift.

The last form of polyaniline to be examined is EB. A somewhat different value of $V_{20}=2.8$ $\mathrm{eV}$ is again needed for a good description of the ground state absorption. In addition we have to modify the last on-site term in (1) to reflect a different site energy on the protonated(-2.5 $\mathrm{eV})$ versus unprotonated $(-1.5 \mathrm{eV})$ nitrogen sites[16]. Even with those changes a satisfactory ground state absorption is obtained only if we follow the conventional assumption that the amine groups(-NH-) appear in adjacent pairs regularly. The calculated absorption curve of the ground state of EB does seem to agree with the experimental data[15]. For the model used here the dominant elementary excitaions are bipolarons[14] again. The photoinduced absorption and bleaching due to an oppositely charged bipolaron pairs are shown in Fig. 4. That may explain the photoinduced absorption features seen[17] at about $0.9 \mathrm{eV}$ and $3 \mathrm{eV}$ after Coulomb shift. Considering the simplicity of the tight-binding model this is a remarkable fit for a complex material such as EB.

In summary, we have attempted to understand the low-lying excitations of polyaniline within a simple tight binding model. This is a minimum model involving both bond order and ring torsional order parameters. We have demonstrated that with our theoretical results, many of the observed optical and magnetic properties of the localized excitations can be interpreted

and correlated. In particular, we have elucidated the role played by the neutral solitons. As such the theoretical model forms an important basis for further work.

\section{ACKNOWLEDGEMENTS}

This work was initiated during a sabbatical leave of W. P. Su at the Ohio State University. He thanks the Department of Physics at OSU, especially Professor J. W. Wilkins, for their 
support and hospitality. He also thanks R. McKenzie, J. M. Leng, K. Coplin, K. R. Cromack, and K. Kim for useful discussions. This work was partially supported by the Robert A. Welch Foundation, by DARPA through a contract monitored by ONR and by the DOE-Basic Energy Sciences, Division of Materials Research.

\section{References}

[1] W. P. Su , J. R. Schrieffer, and A. J. Heeger, Phys. Rev. B22, 2099(1980).

[2] J. M. Ginder and A. J. Epstein, Phys. Rev. B41, 10674(1990).

[3] M. C. dos Santos and J. L. Brédas, Phys. Rev. Lett. 62, 2499(1989); ibid. 64, 1185(1990).

[4] R. Ball, W. P. Su, and J. R. Schrieffer, J. Phys. (Paris) C3, 429(1983).

[5] J. M. Leng, J. M. Ginder, R. P. McCall, H. J. Ye, A. J. Epstein, Y. Sun, S. K. Manohar, and A. G. MacDiarmid, Synth. Met. 41-43, 1311(1991).

[6] J. M. Ginder and A. J. Epstein, Phys. Rev. Lett. 64, 1184(1990).

[7] J. P. Pouget, M. E. Jozefowicz, A. J. Epstein, X. Tang, and A. G. MacDiarmid, Macromolecules 24, 779(1991).

[8] A. J. Heeger, S. Kivelson, J. R. Schrieffer, and W. P. Su, Rev. Mod. Phys. 60, 781(1988).

[9] K. Coplin, Y. Sun, S. K. Manohar, A. G. MacDiarmid, S. Jasty, and A. J. Epstein, these proceedings; and to be published.

[10] K. R. Cromack, A. J. Epstein, J. G. Masters, Y. Sun, and A. G. MacDiarmid, Synth. Met. 41-43, 641(1991); and to be published.

[11] S. Long, K. R. Cromack, Y. Sun, A. G. MacDiarmid, and A. J. Epstein, these proceedings; and to be published.

[12] J. M. Leng, J. M. Ginder, R. P. McCall, H. J. Ye, Y. Sun, S. K. Manohar, A. G. MacDiarmid, and A. J. Epstein, Phys. Rev. Lett. 68, 1184(1992).

[13] R. MacKenzie, J. M. Ginder, and A. J. Epstein, Phys. Rev. B44, 2362(1991).

[14] B. Sjogren and S. Stafstrom, J. Chem. Phvs. 88, 3840(1988).

[15] R. P. McCall, J. M. Ginder, J. M. Leng, H. J. Ye, S. K. Manohar, J. G. Masters, G. E. Asturias, A. G. MacDiarmid, and A. J. Epstein, Phys. Rev. B41, 5202(1990).

[16] H. Y. Choi and E. J. Mele, Phys. Rev. Lett. 59, 2188(1987).

[17] M. G. Roe, J. M. Ginder, P. E. Wigen, M. Angelopoulos, and A. G. MacDiarmid, Phys. Rev. Lett. 60, 2789(1988). 


\section{DISCLAIMER}

This report was prepared as an account of work sponsored by an agency of the United States Government. Neither the United States Government nor any agency thereof, nor any of their employees, makes any warranty, express or implied, or assumes any legal liability or responsibility for the accuracy, completeness, or usefulness of any information, apparatus, product, or process disclosed, or represents that its use would not infringe privately owned rights. Reference herein to any specific commercial product, process, or service by trade name, trademark, manufacturer, or otherwise does not necessarily constitute or imply its endorsement, recommendation, or favoring by the United States Government or any agency thereof. The views and opinions of authors expressed herein do not necessarily state or reflect those of the United States Government or any agency thereof. 

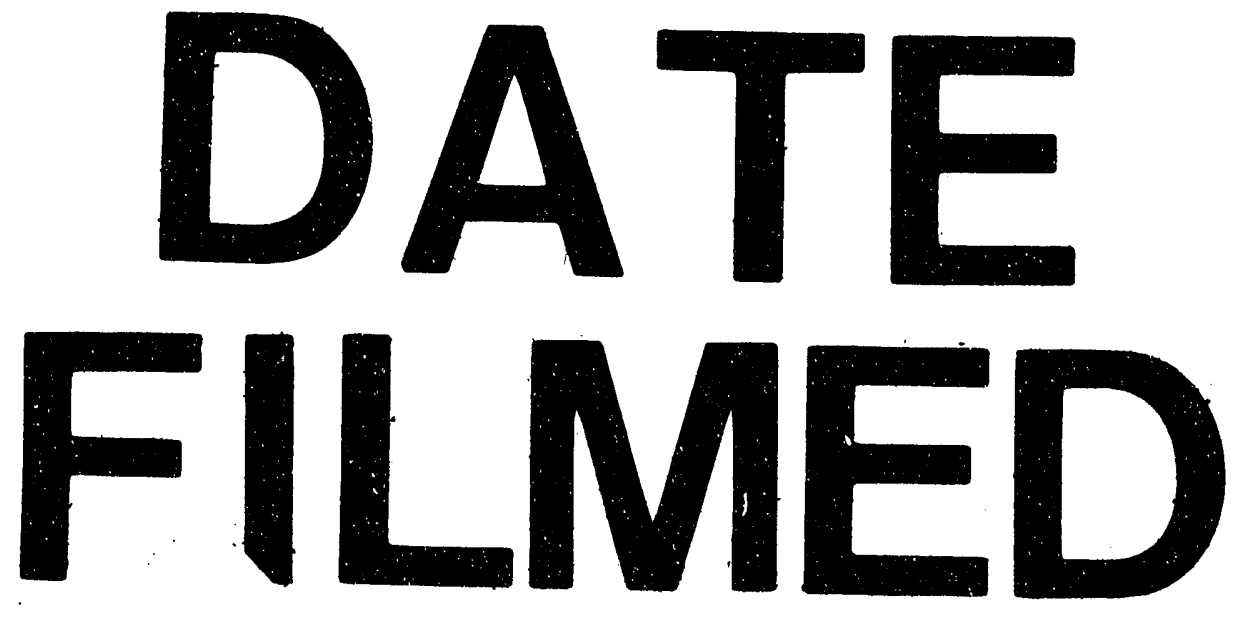

$\cdots$

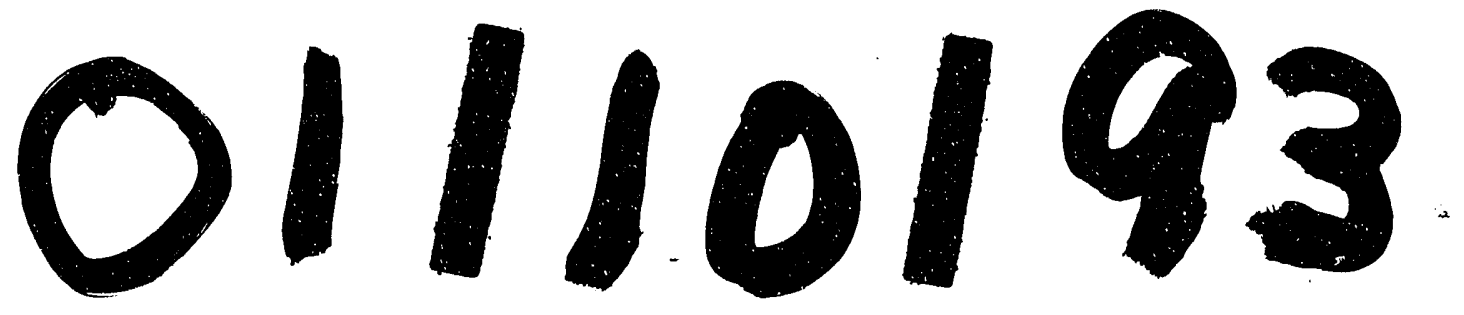


